\title{
GE Healthcare technology column for Critical Ultrasound Journal
}

\author{
Michael Blaivas
}

Published online: 26 September 2009

(C) Springer-Verlag 2009

Several years ago when WINFOCUS first entered negotiations with Springer for publication of a journal, we realized that a peer-reviewed, quality publication would be an expensive undertaking and this would be difficult for a fledgling society. Fortunately, GE Healthcare was interested in supporting our project and provided an unrestricted grant to support 1 year of journal publications. This would allow us to get the journal off the ground and gain momentum that otherwise would have taken additional years to achieve. GE Healthcare specifically stipulated, as did WINFOCUS, that there would be no editorial input or control of the journal. This allowed us to remain unbiased and to provide a dedicated international forum for ultrasound care. We have also asked GE Healthcare for another contribution to the journal: a technical column describing ultrasound innovations, scientific developments related to ultrasound and engineering insights into the design of current and future ultrasound machines. The goal was to provide our readers an engineering insight into how ultrasound works and what new features may eventually be helpful to us at the patient's bedside.

This is the first and introductory column by GE Healthcare and will be followed by other technical ones. We believe that it will give added educational information to our readers without compromising on the editorial integrity. We are grateful to GE Healthcare for agreeing to provide these articles.

\footnotetext{
M. Blaivas $(\square)$

Editor-in-Chief

Department of Emergency Medicine,

Northside Hospital Forsyth,

Cumming, GA 30040, USA

e-mail: mike@blaivas.org

M. Blaivas

Editor-in-Chief

University of South Carolina Medical School,

Columbia, SC, USA
} 